\title{
Políticas y tecnologías de radares: el caso de Argentina en el contexto Latinoamericano
}

Sofia Foladori-Invernizzi ${ }^{1}$

Recibido: 28/02/2021; Aceptado: 05/05/2021

Cómo citar: Foladori-Invernizzi, S. (2021). Políticas y tecnologías de radares: el caso de Argentina en el contexto Latinoamericano. Revista Hipertextos, 9 (15), 143-156. DOI: https://doi.org/10.24215/23143924e032

Resumen. El desarrollo de los radares se remonta a unos años antes de la Segunda Guerra Mundial, y su nombre deriva de un acrónimo: Radio Detection and Ranging. En este trabajo, tomando el caso de la tecnología de radares en Argentina, evidenciamos algunos desafíos para alcanzar autonomía tecnológica en países periféricos. En Argentina, la producción de radares nacionales ocurrió mucho después que en los países industrializados y hasta entrados los años 2000 las políticas de radarización no fueron efectivas, y muchos de los planes iniciados fueron descontinuados. Mismo tarde en el contexto internacional, Argentina se destaca por el pionerismo en la tecnología de radares en el contexto latinoamericano. Examinando el caso de los radares en Argentina podemos discutir algunas cuestiones centrales en el área de la ciencia y la tecnología, tales como la transferencia tecnológica, la dependencia científica y la tecnología dual. Dada la característica dual de los radares, las cuestiones geopolíticas en distintos momentos históricos constituyen un punto central en su desarrollo. A partir del análisis documental y de literatura, evidenciamos la efectiva importancia y destacado rol de Argentina en el contexto latinoamericano respecto de la tecnología de radares aéreos que pasó, en pocos años, de tener una pequeña porción del territorio nacional cubierto por radares a cubrir prácticamente toda el área nacional, cambio que ocurrió después del inicio de la fabricación nacional de radares. También se destaca la importancia de la autonomía tecnológica en Argentina y el papel central jugado por la empresa INVAP. Finalmente, es necesario reflexionar sobre la necesidad de un apoyo continuo y sista aemático del Estado para hacer sustentable un proyecto de autonomía tecnológica como el analizado. En áreas de alta tecnología, los logros conseguidos por países periféricos están siempre amenazados por la acelerada dinámica de innovación liderada por los países centrales.

Palabras clave: tecnología dual, tecnología de radares, políticas de radarización, dependencia científico tecnológica.

Sumario. 1. Introducción. 2. El caso argentino y su contexto histórico. 3. La dependencia científicotecnológica y la tecnología dual. 4. Conclusiones.

\footnotetext{
1 Bióloga, Estudiante de Maestria en Ciencia, Tecnologia e Innovación en la Universidad Nacional de Rio Negro. Contacto: sofiafoladori@gmail.com
} 


\section{Radar policies and technologies: the case of Argentina in the Latin American context}

Abstract. The development of radars dates back to a few years before World War II, and its name derives from an acronym: Radio Detection and Ranging. In this work, taking the case of radar technology in Argentina, we present some challenges to achieve technological autonomy in peripheral countries. In Argentina, the production of national radars occurred much later than in industrialized countries. Until the early 2000s, the radarization policies in Argentina were not effective, and many of the plans that started were discontinued. However, even late in the international context, Argentina stands out as pioneer on radar technology in the Latin American context. Examining the case of radars in Argentina, we can discuss some crucial issues in the area of science and technology, such as technology transfer, scientific dependence and dual technology. Given the dual characteristic of radars, geopolitical issues and historical moments constitute a central point in their development. From the analysis carried out, we can conclude the importance and prominent role of Argentina in the Latin American context with respect to radar technology. In a few years they passed from having a small portion of the national territory covered by radars to covering practically the entire national area, a change that occurred only after the beginning of national production of radars. The importance of technological autonomy in Argentina and the central role played by the INVAP Company are also highlighted. Finally, it is necessary to reflect on the need for continuous and systematic support from the state to allow for technological autonomy in cases such as the one analyzed in this paper. In high-tech areas, the achievements of peripheral countries are threatened by the accelerated dynamics of innovation led by central countries.

Keywords: dual technology, radar technology, radarization policies, scientific technological dependence

\section{Politicas e tecnologías de radares: o caso da Argentina no contexto Latinoamericano}

Resumo. O desenvolvimento de radares remonta a alguns anos antes da Segunda Guerra Mundial, e seu nome deriva de uma sigla: Radio Detection and Ranging. Neste trabalho, tomando o caso da tecnologia de radar na Argentina, mostramos alguns desafios para alcançar a autonomia tecnológica em países periféricos. Na Argentina, a produção de radares nacionais ocorreu muito mais tarde do que nos países industrializados, e até o início dos anos 2000 as políticas de radarização não foram efetivas e muitos dos planos iniciados foram interrompidos. Porém, mesmo tardiamente no contexto internacional, a Argentina se destaca pelo pioneirismo da tecnologia de radar no contexto latino-americano. Examinando o caso dos radares na Argentina, podemos discutir alguns temas centrais na área de ciência e tecnologia, como transferência tecnologica, dependência científica e tecnologia dual. Dad o duplo carater dos radares, as questões geopolíticas e os momentos históricos constituem um ponto central em seu desenvolvimento. A partir da análise realizada, podemos concluir a importância efetiva e o papel de destaque da Argentina no contexto latino-americano no que diz respeito à tecnologia de radar aéreo, que passou, em poucos anos, de ter uma pequena porção do território nacional coberto por radares a cobrir praticamente toda a área nacional, mudança ocorrida após o início da fabricação nacional de radares. Destaca-se também a importância da autonomia tecnológica na Argentina e o papel central da empresa INVAP. Por fim, é necessário refletir sobre a necessidade de apoio contínuo e sistemático do Estado para casos de autonomia tecnológica como o aqui analisado. Nas áreas de alta tecnologia, as conquistas dos países periféricos são sempre ameaçadas pela dinâmica acelerada da inovação liderada pelos países centrais.

Palavras-chave: tecnologia dual, tecnologia de radar, políticas de radarização, dependência científica e tecnológica 


\section{Introducción}

El inicio del desarrollo de los radares se remonta a unos años antes de la Segunda Guerra Mundial, y su nombre deriva de un acrónimo: Radio Detection and Ranging (Quiroga \& Aguiar, 2016; Vassallo, 2008). Estos estaban siendo estudiados tanto por instituciones gubernamentales como por universidades y grupos autónomos en diversos países de Europa y en Estados Unidos. En 1938 se hizo el primer radar pulsado centimétrico, el cual permitió localizar barcos y aviones (Susskind, 1994).

Bajo la presión del conflicto bélico, en poco tiempo y antes del fin de éste, los países industrializados ya habían desarrollado sus propios radares. Estados Unidos, Reino Unido, Francia, Alemania, Italia, Japón y Rusia, todos contaban con sus radares de producción nacional. A pesar de registros de cierto nivel de transferencia tecnológica entre algunos de los países citados, la mayor parte del desarrollo fue interno de cada nación (Susskind, 1994). Según este autor es común, una vez que la ciencia y tecnología llegan a satisfacer todos los prerrequisitos necesarios para innovaciones, que ocurran desarrollos de manera simultánea en distintos lugares, como fue el caso de los radares.

Los radares utilizan tecnologías digitales, las cuales abarcan mucho más que el sentido etimológico de su término (digitalis - dedo). Por tecnología digital se entiende la captación, presentación, sistematización, transporte, combinación y almacenamiento de datos mediante bits. Este tipo de sistemas que conecta los diversos dispositivos físicos entre sí codificándoles digitalmente se conocen como ciberfísicos (Prime Faraday, 2002).

En la base técnica del proceso de tecnología digital están, hoy en día, los Mems (micro electrical mechanical sistemas) - o Moems cuando utilizan procesos ópticos, o Nems cuando son en escala nanométrica - dispositivos diminutos que incorporan sensores, actuadores, y controladores formando sistemas inteligentes de información. Los sensores captan las fuerzas de la materia y la convierten en señales eléctricas. Mediante los Mems puede ser identificada y procesada la fuerza que manifiestan, sea hidráulica, neumática, magnética, motriz, etc., y transmitida a los controladores y al sistema en su conjunto (Prime Faraday, 2002).

Los radares para aeronáutica utilizan ondas electromagnéticas, enviando señales cuyo eco, viajando a la velocidad de la luz, permite medir objetos, tamaños, cantidades, velocidades de movimiento, localización, distancia, y otras características (Prime Faraday, 2002). Los Mems para uso en radares y aviones son muy diversos. Existen mercados específicos para Mems de presión, temperatura, fuerza, torsión, velocidad, posición y desplazamiento, nivel, cercanía, captación de flujos, acelerómetros, giroscopios, gps, etc. (Markets\&Markets, 2020).

Existen dos tipos de radares para el control aéreo: los radares primarios y los secundarios. Los radares primarios permiten obtener informaciones sobre la localización del avión - u otro objeto en el espacio aéreo - actuando mediante la emisión de onda y vuelta de esta al iluminar con un objeto. Vale destacar que consiguen identificar cualquier objeto, mismo que este no quiera ser identificado, pues no dependen de ninguna otra señal. En contraste, los radares secundarios se comunican con la aeronave mediante el auxilio de un Transponder. Así, la aeronave también tiene una estación radioeléctrica, permitiendo la comunicación entre las estaciones de radar (Omnisys, 2017; Vassallo, 2008). Hay una diferencia esencial entre esos dos tipos de radares. Los primarios, como ya mencionado, son independientes de otra señal y, por ende, se encajan en un contexto militar además de civil. Los radares primarios pueden subdividirse en otros dos grupos, los radares 2D y los 3D. Esta distinción está relacionada a cuantas dimensiones capta el radar. 
Los $2 \mathrm{D}$ adquieren información de dos dimensiones. Estos miden el acimut, que permite obtener información de la distancia y de la ubicación en el plano. Los radares 3D, en cambio, además de ubicación en el plano y distancia, consiguen también obtener la altura del objeto (Alonso, 2018; González, 2017).

En este trabajo, tomando el caso de la tecnología de radares en Argentina, evidenciamos algunos desafíos para alcanzar autonomía tecnológica en países en desarrollo. En la segunda sección, siguiendo a esta introducción, presentamos, en una línea del tiempo, las políticas para el desarrollo de radares en la Argentina, diferenciando un período de radarización basada en la importación de tecnología y otro en que se desarrolla la producción nacional. En la tercera sección examinamos la dependencia científico-tecnológica, abordando cuestiones como la transferencia de tecnología y la dependencia científica y discutimos la característica dual de esta tecnología. En esa misma sección, consideramos la necesidad de un conjunto de políticas públicas convergentes para asegurar la sostenibilidad de la tecnología nacional de radares. Finalmente, presentamos las conclusiones del trabajo.

\section{El caso argentino y su contexto histórico}

En Argentina, la producción de radares nacionales ocurrió mucho después que en los países industrializados. Hasta los años 2000, Argentina sólo disponía de radares importados, que cubrían una pequeña parte del área nacional. A pesar de diversas políticas para la producción de radares y para el control aéreo argentino, hasta entrados los años 2000, éstas no fueron efectivas y muchos de los planes iniciados fueron descontinuados (Quiroga, 2018; Quiroga \& Aguiar, 2016). En la imagen abajo (Figura 1) podemos ver una línea del tiempo con algunos de los principales momentos clave para el desarrollo de la tecnología de radares en Argentina.

Figura n ${ }^{\circ}$ 1. Línea del tiempo del desarrollo de la tecnología de radares en Argentina.

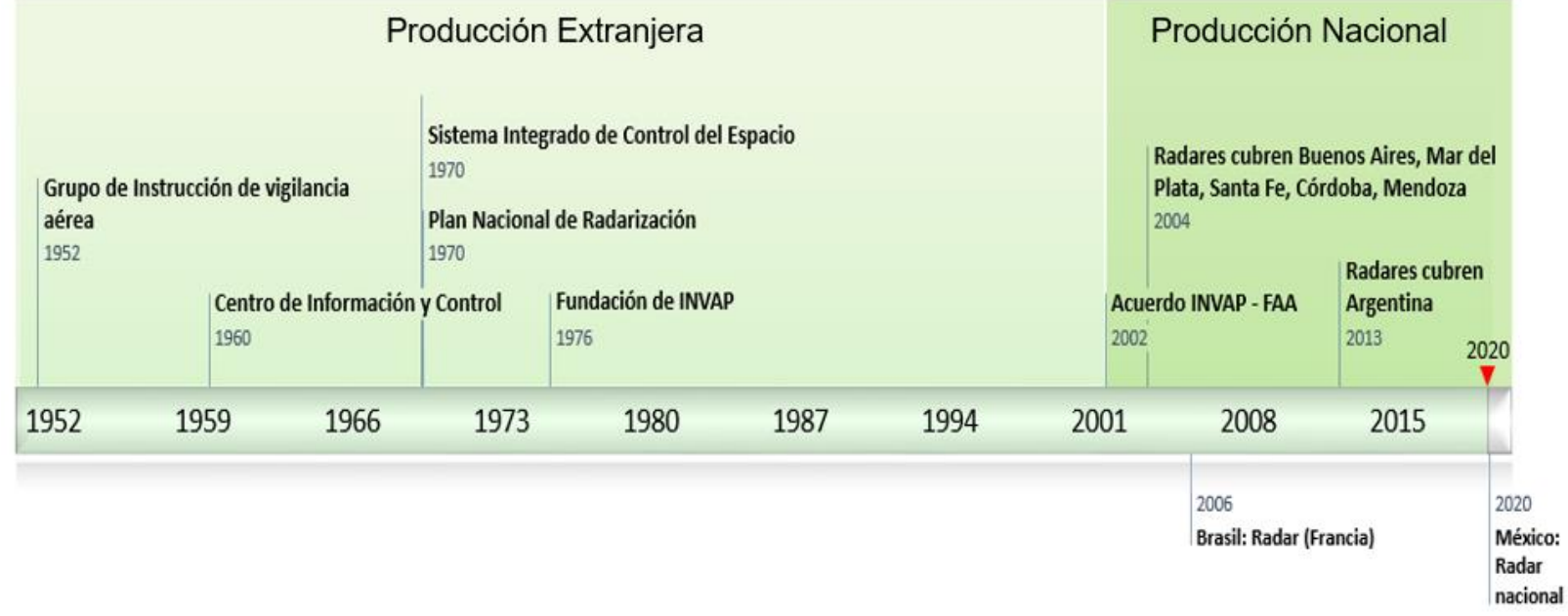

Fuente: la autora, con base en Quiroga (2018).

Leyenda: En la figura es posible ver los avances que obtuvo Argentina desde 1950 para el desarrollo de radares nacionales. La línea está dividida en dos momentos, antes y después de la

presencia de tecnología nacional. Además, para fines de comparación, es posible observar cuando fueron fabricados radares en Brasil y en México. 
Terminada la Segunda Guerra Mundial, Argentina poseía radares, de origen importado, alrededor de la Ciudad de Buenos Aires para defensa, vigilancia y control aéreo. A partir de los años 50 tienen inicio las políticas para la radarización de Argentina. En 1952 se forma el Grupo de Instrucción de Vigilancia Aérea y, algunos años después, en 1960, el Centro de Información y Control (Quiroga, 2018).

En los años 70 es implementado un Plan Integrado de Radarización para Argentina, el Proyecto Sistema Integrado de Control del Espacio Aéreo (SICEA). Este proyecto, como ya lo dice el nombre, consistía en una política integrada, abarcando tanto los objetivos de aviación civil como los militares de defensa del territorio. Empero, este proyecto es descontinuado algunos años más tarde, en 1984. Más de diez años después del SICEA, en 1996, surge otra política para los radares, el Plan Nacional de Radarización, proyecto que nunca llegó a ser ejecutado (Quiroga, 2018).

Aún en esta fase, tiene lugar, en 1976, la fundación de la empresa tecnológica Argentina INVAP - Investigaciones Aplicadas (INVAP, 2020), creada como una sociedad del Estado a partir de un convenio entre la Comisión Nacional de Energía Atómica (CNEA) y el Gobierno de la Provincia de Río Negro (Potenze, 2017). Esta tendría una importancia significativa para el desarrollo de los radares, aunque, en su inicio, la empresa se dedicaba a la tecnología nuclear y posteriormente - también de satélites. Entrados los años 2000 esta empresa busca nuevas aberturas de mercado y, con eso, entra en la tecnología de radar al ofrecerle a la Fuerza Aérea Argentina (FAA) el desarrollo de un radar nacional en 2002. El año que prosigue, la FAA firma un acuerdo con INVAP para la producción de un prototipo de radar secundario, y luego otros diez radares (Quiroga, 2018).

En este momento ocurre un giro decisivo para la nacionalización de la tecnología de radares, cuando se crea, en 2004, el SINVICA, Sistema Nacional de Vigilancia y Control Aeroespacial, en el cual consta, explícitamente, la priorización de la producción nacional. Este destaque a la industria nacional no estaba presente en las dos tentativas anteriores de radarización, que se basaban en una completa dependencia tecnológica (Quiroga, 2020). Además, en este momento, Argentina ya poseía cobertura de radares secundarios en las áreas con mayor tránsito aéreo, abarcando las regiones alrededor de Buenos Aires, Mar del Pata, Santa Fe, Córdoba y Mendoza (Quiroga, 2018; Vassallo, 2008). En menos de diez años, Argentina pasó a tener casi todo el territorio nacional cubierto por radares (Quiroga, 2018), sin embargo, debe mencionarse que estos radares no eran de producción nacional.

En 1995 la empresa norteamericana Lockheed Martin adquirió una empresa pública en Córdoba para la fabricación de aviones militares. En 2000, o sea, pocos años antes de la creación del SINVICA, el gobierno firmó un nuevo contrato con la Lockheed Martin que envolvía 230 millones de dólares y la producción de diversos productos militares para actualizar la flota aeronáutica, incluyendo un nuevo radar AN-APG-67(V)4 (Defence-aerospace, 2002; Lorenzo, 2002). Como menciona Quiroga (2018), hay una fuerte relación entre los avances tecnológicos en el área de radar y en la aeronáutica, pues estos se deben adecuar a las diferentes características de los aviones. Es entonces importante destacar el contrato con la Lockheed Martin, pues si la producción de aviones militares sigue siendo extranjera, ¿hasta qué punto puede haber una efectiva independencia y producción suficiente y eficiente de radares de producción nacional?

Se nota, en esta breve revisión histórica, que en Argentina hubo diversas tentativas fallidas para la creación de un sistema nacional del control aéreo. Sin embargo, como argumenta Quiroga (2018), todas ellas tuvieron un impacto importante en el posterior desenvolvimiento del 
SINVICA. En 2008, Argentina consiguió por primera vez realizar la detección de una aeronave a partir del radar diseñado y producido por la empresa rionegrina INVAP, permitiendo, así, la entrada de Argentina al grupo de países que poseen desarrollo proprio de tecnología de radares (Quiroga \& Aguiar, 2016).

No obstante el avance del desarrollo de radares nacionales y la mayor cobertura del territorio, el sistema de estos todavía se encontraba debilitado al inicio de los años 2000. En 2000 hubo un incendio en la torre de control del aeropuerto de Ezeiza en Buenos Aires, que evidenció la falta de equipamientos de emergencia. Algunos años después, en 2007, ocurrió una tormenta eléctrica en la ciudad de Buenos Aires que dañó el sistema de radar, también en Ezeiza, dejando el servicio de control de aviones apenas por medio convencional mediante comunicación por radio con la torre (Vassallo, 2008).

Argentina se destaca por el pionerismo en la tecnología de radares. Fue el primer país de Sudamérica en tener radares para el control del tráfico aéreo, en 1948, siendo estos importados (González, 2014 apud.; Quiroga \& Aguiar, 2016). Al comparar con otros países de América Latina, el desarrollo de la tecnología nacional de radares en Argentina fue temprano. En Brasil, la producción de radares es realizada por una multinacional francesa, Thales, que compró una empresa brasileña, la Omnisys, en 2006 e inició la fabricación de radares de tráfico aéreo. A pesar de que la Omnisys había sido pionera - en términos de Latinoamérica - en el desarrollo de un radar meteorológico en 2005, la producción de radares de tránsito aéreo apenas comenzó después de la adquisición por la empresa Francesa, dando origen al radar LP23SST NG. En el caso de Brasil la transferencia tecnológica fue total (Vasconcelos, 2013), muy diferente de lo observado en Argentina.

Es interesante notar que Brasil es el país con mayor producción mundial de Niobio, un elemento muy utilizado en la fabricación de radares. En 2012, la producción brasilera abarcaba el $93 \%$ de la producción mundial (Aita et al., 2016). Brasil, teniendo predominancia en la explotación de este material fundamental, igual posee una dependencia tecnológica total en la fabricación de un producto que deriva de su utilización, el radar.

En México el desarrollo de radares fue aún muy posterior. En 2020 fue producido el primer radar móvil nacional, el Tzincán, a partir de un proyecto que inició en 2015. Así como en Argentina y en Brasil, el Tzincán fue resultado de un programa con objetivos tanto civiles como militares, englobando tres instituciones mexicanas: el Consejo Nacional de Ciencia y Tecnología (CONACYT), en conjunto con la Secretaría de la Defensa Nacional (SEDENA) y la Secretaría de Marina-Armada de México (SEMAR). Además, México, a diferencia de Argentina, posee todavía grandes lagunas en el control por radar de su territorio nacional, con grandes zonas grises en el sur y dejando el norte, en gran parte, bajo control de radares de los Estados Unidos (Medina, 2020). El país cuenta con apenas un tercio del área nacional con cobertura área (Guevara, 2020). En parte, el desarrollo de un radar móvil por México es importante para poder moverlo conforme las necesidades en distintos momentos, dada esta escasa cobertura actual del espacio aéreo nacional.

En todo caso, en los tres países, los radares fueron desarrollados con la intención de integrar tanto el tráfico aéreo civil cuanto el control militar. Aquí se destaca la función dual de la tecnología de radar, que es producida ya previendo ambos fines: civil y militar. En el proprio sito de INVAP, los radares se encuentran dentro de la categoría de "defensa, seguridad y ambiente" 
(INVAP, 2020), ya haciendo alusión a su importancia militar, además de su inicio vinculado a las FAA (Quiroga, 2018), aludiendo también a usos civiles como el monitoreo ambiental.

\section{La dependencia cientifico-tecnológica, la tecnología dual y reflexiones sobre las políticas vinculadas a la tecnología de radares}

El examen del caso de los radares en Argentina nos lleva a la discusión de algunas cuestiones importantes en el área de la ciencia y la tecnología, tales como la transferencia tecnológica, la dependencia científica y la tecnología dual.

Según Diamand (1976), el desarrollo industrial latinoamericano fue respaldado con tecnología importada, lo que configuró una traba a su desarrollo. La elección entre la producción de tecnología local o la importación de tecnología depende de diversos contextos interdependientes dentro de la economía y de las políticas gubernamentales. De acuerdo con Sábato (2004) el desarrollo de una estructura científicotecnológica y productiva, en las condiciones de países en desarrollo, requiere de la triple interacción entre distintas instancias: la infraestructura científico-tecnológica, el gobierno y la estructura productiva para el desarrollo de tecnología. En este esquema, posteriormente conocido como Tríangulo de Sábato, es el Estado quien adopta un papel clave en el diseño e implementación de la política con el fin de crear la necesaria trama de interacción institucional, tecnológica y económica. Toledo (2019) expone que la distancia entre los países de América Latina y los países centrales respecto al hiato tecnológico y productivo empezó a ser significativa a partir de los años 1970, y desde entonces viene aumentando. El aumento de la especialización y la gran explotación de los países periféricos ha empeorado esta situación, llegando a una situación de neocolonialismo, resultado de la división internacional del trabajo, donde se presentan explícitas las relaciones de dependencia y dominación, principalmente en el aspecto tecnológico. El acceso desigual entre centro y periferia no es únicamente material, se extiende también a los recursos humanos y al acceso a conocimiento aplicable productivamente.

En el contexto de la industrialización por substitución de importaciones, debido a los altos costos de producción industrial presentes en los países latinoamericanos, el desarrollo nacional fue promovido mediane la protección de políticas nacionales que fomentasen esta inversión y protegiesen los mercados nacientes (Diamand, 1976). Aún en el contexto de países desarrollados y de mercados mucho más abiertos, tal como argumenta Mazzucato (2017), el papel de los gobiernos ha sido esencial para promover la innovación y crear nuevos mercados mediante el financiamiento a la investigación y el apoyo a empresas innovadoras. Tal ha sido el caso, como muestra la autora, de las tecnologías para la información y la comunicación y de la biotecnología.

En el caso de los radares en Argentina, la acción del Estado para promover la autonomía tecnológica en la producción de radares se evidencia en diversas políticas nacionales a lo largo de los años, pero problemas de financiamiento e insuficiencia de recursos llevaron a la descontinuación de estos programas, manteniendo, así, la utilización de tecnología importada hasta entrados los años 2000. En el caso de Brasil, la fabricación es hecha en territorio nacional, pero es por medio de una empresa multinacional francesa que importa todo el conocimiento, exponiendo la gran dependencia tecnológica existente.

A pesar de sus mayores costos, la tecnología nacional presenta un conjunto de ventajas, pues ésta se adecua mejor a las condiciones y necesidades nacionales, además de poder beneficiarse de materias primas locales. Además, una vez desarrollada la tecnología, es posible la adaptación a futuros cambios y la capacidad de respuesta frente a nuevas exigencias y necesidades (Diamand, 1976). Ciertamente, todo el proceso reposa en el desarrollo de capacidades científicas, de ingeniería y de fuerza de trabajo nacionales.

Hipertextos, Vol. 9, N 15. Buenos Aires, Enero/Junio de 2021 «149 
No obstante estas grandes ventajas, cabe discutir la eficiencia de la tecnología nacional, especialmente en lo que respecta a tecnologías que presentan un gran dinamismo innovativo liderado por los principales países industrializados. En el caso de México, por ejemplo, poco antes del inicio del proyecto del radar nacional, el gobierno consideró también adquirir radares extranjeros, lo cual no ocurrió debido a los altos costos de compra. El radar mexicano, terminado en 2020 , permite un alcance de $74 \mathrm{~km}$ mientras que las alternativas importadas considerados por México tienen mucho más que el doble de alcance. El radar Thales francés (la misma empresa que actúa en Brasil), tiene un alcance de $460 \mathrm{~km}$, un radar fabricado en los Estados Unidos alcanza $445 \mathrm{~km}$ y un radar español tiene 330km de alcance (Guevara, 2020). Esto nos lleva a cuestionar que reconociéndose las ventajas de la tecnología nacional, pues genera independencia tecnológica, se presenta el problema de la menor eficiencia y si esta llega, efectivamente, a sustituir la tecnología importada.

En el caso de Argentina, si tomamos el Radar Primario 3D de Largo Alcance RP3DLA, producido en 2005, este tiene una gran competencia en el mercado internacional, comparable a los radares franceses, norteamericanos y españoles, alcanzando $400 \mathrm{~km}$ (Alonso, 2018). En relación a la competencia de los radares también hay otros dos puntos importantes: la altura y la capacidad de traslado. El radar mexicano, por ejemplo, tiene la altura considerada estándar de $30.000 \mathrm{~m}$, así como el argentino, y compensa su falta de alcanceen kilómetros por el hecho de ser móvil (Guevara, 2020).

Otra cuestión importante a ser considerada al tratar de radares es la función dual de estas tecnologías, que son creadas tanto con fines militares como civiles, como ya se ha mencionado anteriormente. La tecnología de radares, teniendo su inicio antes de la Segunda Guerra Mundial, avanzó decisivamente durante el conflicto bélico (Alic, 1994; Susskind, 1994). Desarrollada como una tecnología militar pasó luego a ser utilizada con función civil, en un proceso de spillover, de una esfera para otra. Empero, una vez desarrollada la tecnología inicial, esta pasó a tener un carácter inherentemente dual, previendo desde su desarrollo hasta su utilización una función en ambas esferas (Alic, 1994; Cowan \& Foray, 1995), como es observado en muchas de las políticas que contienen títulos como "integrada".

Alic (1994), por ejemplo, argumenta que prácticamente todas las tecnologías pueden ser consideradas duales. Partiendo de ese principio, el autor expone las grandes ventajas de la tecnología dual. Como la tecnología es aplicable en diversas esferas, hay una reducción de costos y desarrollo más eficiente si se produce desde el inicio con un fin dual. Sin embargo, las tecnologías dichas duales tienen un gran aporte miliar y de defensa y, por ende, materializan objetivos militares de control y de defensa, que se efectivizarían también en su uso civil. Puede cuestionarse que si determinadas tecnologías no tuviesen en su diseño esta influencia militar, y su diseño fuese más específicamente direccionado para el uso civil, podrían tener un mejor desempeño.

La proliferación de tecnologías de uso dual está fuertemente vinculada al fin de la Guerra Fría, en 1989. Al terminar ésta, los recursos destinados en los presupuestos nacionales al ramo militar y de defensa se redujeron, notoriamente en los Estados Unidos y algunos países europeos. Surgió, así, el desafío de mantener el ejército y los desarrollos militares con menos recursos, además de mantener un complejo sistema de industrias que producían tecnologías para uso militar. Este cambio geopolítico, que afectó a todo el mundo, llevó a la concepción del término tecnología-dual, y su discusión en la esfera económica y política (Alic, 1994; Brandt, 1994). Es 
importante notar aquí que tecnologías producidas con diversos fines y spillovers en la industria tecnológica siempre existieron, pero estos pasan a tener una centralidad nueva, al permitir la sobrevivencia del complejo industrial-militar, ahora destinado a la producción de tecnología dual. A partir de este momento histórico la tecnología dual pasa a ser punto de discusión de políticas de desarrollo tecnológico.

Otra cuestión relevante en el ámbito geopolítico que afectó la tecnología de radares fue, sin duda, la necesidad de aumentar la seguridad del espacio aéreo debido al aumento del tráfico de drogas a partir de los años noventa. Para eso son fundamentales los esfuerzos por nacionalizar la producción de radares y de cubrir el territorio nacional. Las zonas no abarcadas por radares se presentan como una facilidad a la entrada de droga en esas regiones. Así, el combate al tráfico de drogas se configura como otra razón para la necesidad e importancia del control y carácter nacional de las tecnologías e información de los radares. Una tecnología nacional supone también que la información sea controlada nacionalmente. Tomando por ejemplo la situación de México, en toda la frontera norte con Estados Unidos, área estratégica para el control del tráfico de drogas, el espacio aéreo está controlado por radares de Estados Unidos, los cuales les envían - o no - la información adquirida (Medina, 2020).

Debido a la complejidad científico-tecnológica de los sistemas de radares, su vinculación con asuntos geopolíticos y la función dual que estos poseen, las políticas públicas específicas para estimular su fabricación nacional en países en desarrollo parecen no ser suficientes para englobar un tema tan amplio y complejo. Son necesarias además de políticas de fomento para la radarización, otras políticas sectoriales o vinculadas que se integren a esos planes de desarrollo, auxiliando en cuestiones más específicas.

De entre muchas otras políticas, algunas están directamente relacionadas con la sustentabilidad de la I\&D en radares. Este es el caso, por ejemplo, de políticas vinculadas a la propiedad intelectual. En la actualidad todas las innovaciones tecnológicas están registradas como propiedad intelectual (Piñeiro, 2015). Las patentes y otras formas de Propiedad Intelectual se han convertido en un creciente mercado a nivel internacional. Desde finales de los años ochenta y principios de los noventa la cantidad de patentes registradas en las diversas oficinas se ha incrementado año con año a un ritmo mucho mayor que en década anteriores. Las patentes se consideran un indicador de innovación y desarrollo tecnológico (OMPI, 2010). Pero, registrar una patente no significa necesariamente una ventaja económica. Existe una amplia discusión sobre las ventajas o desventajas de las patentes en el desarrollo económico (Madies et al., 2014).

En el caso de los radares de tráfico aéreo argentinos de INVAP, algunos de estos están patentados, pero quien asume la propiedad de derechos y patentes y la dirección del proyecto es la Dirección General de Fabricaciones Militares (DGFM) según los requerimientos técnicos de la FAA (Piñeiro, 2015). Así, las patentes son de carácter nacional pero, en lugar de estar vinculadas a una empresa provincial están bajo el dominio militar, destacando explícitamente el carácter dual de estas tecnologías, con énfasis en su importancia prioritaria militar.

Dado, además, el alcance internacional de la empresa, que ya ha realizado transferencia de tecnología a varios países, como es el caso últimamente de Holanda, es posible que surja la necesidad del registro de patentes allende Argentina (Caillaud \& Méniere, 2014). En el caso del radar 3D, este cumple los requisitos técnicos y las normas para poder exportar sus radares a nivel internacional (Alonso, 2018). Argentina puede, ahora, cambiar de posición a nivel internacional y actuar como exportadora de tecnología en lugar de importar tecnología extranjera. Sin embargo, 
dado al vínculo militar del radar 3D y el acuerdo hecho con la FAA la venta a otro país es difícil (Alonso, 2018).

Otro ejemplo de políticas sectoriales íntimamente ligadas al desarrollo de los radares es la concerniente a la integración de las cadenas de producción y de valor. En la actualidad, gran parte de la producción industrial mundial corresponde a la integración de muchas empresas con sede en diversos países que participan parcialmente en la producción de la mercancía final (Mayer \& Gereffi, 2010). Si tomamos el caso del radar 3D RP3DLAP argentino, se tiene que 80 $\%$ de los componentes son fabricados localmente, lo que evidencia una alta tasa de nacionalización. Cabe indagar si el $20 \%$ restante es efectivamente imprescindible de ser importado o si se podría eventualmente llegar a una producción integralmente nacional. Además, siguiendo la cadena de producción se nota la gran participación de pequeñas y medias empresas (pymes) vinculadas. Hay alrededor de 500 pymes que han participado en el proceso de fabricación de radares, de las cuales 50 tuvieron un rol más significativo (Alonso, 2018). Eso demuestra cómo se está manteniendo la propuesta original del proyecto de radarización de favorecer la industria nacional y, además, consiguiendo crear un tejido productivo con la actuación de pymes y no apenas de industrias grandes ya consolidadas.

Este aspecto del radar RP3DLAP es esencial para la sustentabilidad de la producción y la contínua investigación sobre radares en Argentina u otros países en desarrollo. Solamente una producción a partir de materiales prioritariamente nacionales y con tecnologías relacionadas nacionales, fabricados a partir de recursos humanos locales puede llevar a una independencia tecnológica efectiva y real. López y colaboradores (2018), tratando sobre los satélites argentinos destacan el supuesto de que productos nacionales - como el satélite o el radar - no implican, necesariamente, que todas sus piezas e instrumentos vinculados sean también producidos por una industria nacional, muchos son importados e incorporados a la producción nacional. Empero, se nota como en el caso de los radares argentinos - por lo menos en este último RP3DLAP que es también el más competitivo a nivel internacional debido sus características consiguieron una real e efectiva producción casi integralmente nacional (Alonso, 2018).

Otro factor vinculado a la nacionalización de toda la cadena de producción es que asegura el mantenimiento de los radares instalados, dada la mayor disponibilidad de las piezas de repuesto. Según Abila y colaboradores (2009), la expectativa de vida media de los satélites es de 15 años debido, en gran parte al desgaste de los materiales. Puede suponerse que los radares experimenten un desgaste semejante.

La empresa INVAP, que desarrolla y fabrica los radares argentinos, opera también con la fabricación de satélites. La integración tecnológica entre los radares y los satélites es fundamental para el "comando del espacio", hablando en términos militares. La interconexión de estas tecnologías es favorecida en la Argentina por una empresa única que realiza ambos equipamientos (Abila et al., 2009). Además, el hecho de que INVAP iniciara con la tecnología nuclear y de satélites también facilitó el desarrollo de los radares, dado que algunas de las bases tecnológicas son intercambiables y relacionadas, y los propios satélites utilizan radares como cargas útiles (Alonso, 2018; López et al., 2018). Esto demuestra el papel decisivo de la acumulación de capacidades científico-tecnológicas en las empresas de alta tecnología.

Así, la independencia tecnológica en el área de radares también permite auxiliar la independencia en otras esferas que poseen tecnologías equivalentes y vinculadas. Lleva también estimular la formación de personal calificado y a la construcción de estructuras y laboratorios de 
investigación avanzados (Piñeiro, 2015). Como forma de superación de la dependencia y subordinación de los países periféricos ante los centrales la autonomía tecnológica se presenta como un quesito fundamental. Es necesaria para permitir una autonomía no solo en el ámbito científico-tecnológico, pero también en la política y economía (Toledo, 2019). En México, por ejemplo, para la realización del radar Tzincán, fue realizada en paralelo toda una infraestructura e instalación de laboratorios y softwares idóneos para la fabricación y funcionamiento del radar en cuestión (Medellín, 2020). Esos laboratorios, softwares y personal calificado quedan entonces disponibles para continuar el desarrollo nacional de tecnologías semejantes. En el caso de Argentina el establecimiento de INVAP ya está equipado para distintas tecnologías (Piñeiro, 2015). Así, la producción de radares argentinos direcciona el país hacia una possiblidad de mayor autonomía tecnológica no solo en el área de radares, pero también en otras tecnologías vinculadas, como las tecnologías digitales y satelitales.

Aún otro ejemplo de políticas sectoriales relacionadas a los radares son las que atañen al capital humano. Argentina tiene un antiguo y extenso historial en el desarrollo de las ciencias físicas y químicas, y junto con Brasil y México, se posicionaron después de la Segunda Guerra Mundial estimulando la investigación en energía nuclear para usos pacíficos (Junior et al., 2013). Estas capacidades científicas fueron sin duda esenciales para el éxito de la empresa INVAP, que comenzó investigando en el tema nuclear. Fueron también la base para el boom de la investigación y producción de radares en América Latina con la exitosa experiencia brasileña y mexicana. Estos casos son indicios claros de la necesidad de una especialización sistemática en la formación de recursos humanos calificados con apoyo estatal nacional para garantizar un futuro sustentable. Además, varios desarrollos tecnológicos del presente siglo de carácter facilitador, como es el caso de las nanotecnologías, obligan a que las políticas nacionales de C\&T sean altamente integradoras en su apoyo a trayectorias tecnológicas exitosas (Hurtado et al., 2017).

Aún en el contexto de América Latina es interesante citar la creación, en 2008, de la UNASUR, Unión de las Naciones de América del Sur, con el objetivo de cooperación entre 12 países para asegurar la paz e integrar la seguridad, producción industrial y políticas de investigación e innovación tecnológica entre Argentina, Bolivia, Brasil, Chile, Colombia, Ecuador, Guyana, Paraguay, Perú, Surinam, Uruguay y Venezuela. En el mismo año fue creada, a partir de una iniciativa brasileña y partiendo de la propia UNASUR, el SADC, Consejo de Defensa Sud Americano, con el objetivo de formar una base regional de defensa integrada. Al integrar estos dos proyectos se nota tanto la presencia de la tecnología dual, uniendo políticas y desarrollo de tecnología como defensa regional, cuanto la necesidad de producción de tecnologías nacionales a nivel de Latinoamérica capaces de interactuar entre si (Aita et al., 2016). Apenas teniendo la producción y propiedad intelectual de la tecnología es posible hacer las adaptaciones y adecuaciones necesarias para modificarlas e integrar los sistemas de distintos países.

\section{Conclusiones}

A partir de los temas discutidos en este breve ensayo podemos esbozar algunas conclusiones y cuestionamientos respecto las políticas científicas y tecnológicas de radares en América Latina y, más específicamente, en Argentina. Con un tema como los radares, que son tecnologías altamente duales con claras e importantes funciones en el área militar y civil, las cuestiones geopolíticas y el contexto histórico constituyen un punto central en su desarrollo. Efectivamente, 
el surgimiento y desarrollo del radar estuvo directamente vinculado con la Segunda Guerra Mundial y con la Guerra Fría. El amplio desarrollo de la aviación civil desde los años 1950 y mucho más aceleradamente en las décadas recientes demandó ampliar la innovación en radares para garantizar la seguridad del tráfico aéreo. Después, entrados los años 2000, se plantea la necesidad de control del tráfico de drogas, y junto con eso el aumento de la radarización en Argentina, Brasil y México.

A partir del análisis temporal realizado, podemos concluir la efectiva importancia y destacado rol de Argentina en el contexto Latinoamericano respecto de la tecnología de radares aéreos que pasó, en pocos años, de tener una pequeña porción del territorio nacional cubierto por radares a cubrir prácticamente todaa el área nacional. Este cambio se nota después del inicio de la fabricación nacional de radares.

Es fundamental también destacar la importancia de la autonomía tecnológica en Argentina y el papel central jugado por la empresa INVAP, que inició con investigación nuclear, luego satélites y radares. Esas tecnologías y avances permitieron la capacitación de personal y la creación de la infraestructura necesaria para el desarrollo y mejoramiento de esas tecnologías y la posibilidad de aumentar la investigación sobre otras áreas afines. Sumado a eso, el hecho de que $80 \%$ de los materiales provengan de Argentina y de la gran actuación de pymes en la cadena productiva demuestra que la producción es efectivamente nacional y con alta independencia tecnológica. A esto podemos agregar la presencia de patentes de los productos realizados, pero aquí cabe la consideración de que la patente es propiedad militar y no directamente de la empresa provincial desatacando, nuevamente, el carácter dual y altamente militar de la tecnología de radares.

Finalmente, es necesario reflexionar sobre la necesidad de un apoyo continuo y sistemático del Estado, mediante un conjunto de políticas públicas, para hacer sustentable un caso de autonomía tecnológica como el analizado.

\section{Referencias}

Abila, F., Martins, J. \& Cepik, M. (2009). Armas estratégicas e poder no sistema internacional: O advento das armas de energia direta e seu impacto potencial sobre a guerra e a distribuição multipolar de capacidades. Contexto Internacional, 31(1), 49-83.

Aita, E., Studart, Á. V., \& Oliveira, M. A. G. (2016). Current overview and future perspectives on integrated defense logistics in South America: Opportunities for the regional defence industry. Austral: Brazilian Journal of Strategy \& International Relations, 5(10), 199-228.

Alic, J. A. (1994). The dual use of technology: Concepts and policies. Technology in Society, 16(2), $155-172$.

Alonso, M. (2018). Radar en la tormenta [Universidad Nacional de San Martín]. Agencia TSS. unsam.edu.ar/tss/radar-en-la-tormenta/

Brandt, L. (1994). Defense Conversion and dual-use technology: The push toward civil-military integration. Policy Studies Journal, 22(2), 359-370.

Caillaud, F., \& Méniere, Y. (2014). Strategic intelligence on patents. En Patent markets in the global knowledge economy: Theory, empirics and public policy implications. Madiès, T., Guellec, D., \& Prager, J.-C. 
Cowan, R., \& Foray, D. (1995). Quandaries in the economics of dual technologies and spillovers form military to civilian research and development (Núm. 9509). Department of Economics, University of Western Ontario.

Defence-aerospace. (2002). Lockheed Martin selected to provide radar system for AT-63 Pampa trainer light attack aircraft. Defence-aerospace. http://www.defense-aerospace.com/articlesview/release/3/9449/lockheed-radar-for-at_63-pampa-(apr.-3).html

Diamand, M. (1976). Las posibilidades de una técnica nacional en Latinoamérica (el caso argentino). Estudios Internacionales, 9(34), 10-41.

González, O. F. (2017). Caracteísticas de los distintos tipos de radares. Gaceta Aeronautica. https://www.gacetaeronautica.com/gaceta/wp-101/?p=22789

Guevara, I. (2020). El dios murciélago: Tecnología y radares mexicanos. El Heraldo de México. https://heraldodemexico.com.mx/opinion/2020/6/23/el-dios-murcielago-tecnologiaradares-mexicanos-186672.html

Hurtado, D., Lugones, M., \& Surtayeva, S. (2017). Tecnologías de propósito general y políticas tecnológicas en la semiperiferia: El caso de la nanotecnología en la Argentina. Revista Iberoamericana de Ciencia, Tecnología y Sociedad, 12(34). http://www.revistacts.net/volumen-12numero-34

INVAP. (2020). INV AP - La empresa. INVAP. https://www.invap.com.ar/la-empresa/

Junior, O. F., Hurtado, D. Moreira, I. C., \& Barros, F. de S. (2013). Nuclear wepons in regional contexts: The cases of Argentina and Brazil. An open world: science, technology and society in the light of Niels Bohr's thoughts, Copenhagen.

López, A., Pascuini, P., \& Ramos, A. (2018). Climbing the Space Technology Ladder in the South: The Case of Argentina. Space Policy, 46, 53-63. https://doi.org/10.1016/j.spacepol.2018.06.001

Lorenzo, C. (2002). Lockheed Martin renegotiates terms at Argentina facility. Defense Daily - Dow Jones Factiva, 216(3).

Madies, T., Guellec, D., \& Prager, J.-C. (Eds.). (2014). Patent Markets in the Global Knowledge Economy: Theory, Empirics and Public Policy Implications. Cambridge University Press. https://doi.org/10.1017/CBO9781107110960

Markets\&Markets. (2020). Aircraft Sensors Market by Connectivity, Platform, Sensor, Application, End Use COVID-19 Impact Analysis. Aircraft Sensors Market. https://www.marketsandmarkets.com/Market-Reports/aircraft-sensors-market53630527.html

Mayer, F., \& Gereffi, G. (2010). Regulation and Economic Globalization: Prospects and Limits of Private Governance. Business and Politics, 12(3). https://doi.org/10.2202/1469-3569.1325

Mazzucato, M. (2017). El Estado emprendedor: Mitos del sector público frente al privado. RBA.

Medellín, J. A. (2020). La marina y el ejército mexicanos concluyen su primer radar de vigilancia aérea. defensa.com. https://www.defensa.com/mexico/marina-ejercito-mexicanos-concluyenprimer-radar-vigilancia

Medina, J. (2020, noviembre 29). Entre lo urgente y lo importante: Radares. Aviación21. https://a21.com.mx/index.php/inteligencia-aeroespacial/2020/09/13/entre-lo-urgente-y-loimportante-radares

Omnisys. (2017, junio 22). A Thales apresenta o LP23SST NG, o radar de vigilancia em rota de uso dual civil e militar mais avançado do mundo e já vendido para o Brasil. Omnisys. 
https://www.omnisys.com.br/a-thales-apresenta-o-lp23sst-ng-o-radar-de-vigilancia-em-rotade-uso-dual-civil-e-militar-mais-avancado-do-mundo-e-ja-vendido-para-o-brasil

OMPI. (2010). Indicadores mundiales de propiedad intelectual. Organización Mundial de la Propiedad Intelectual.

http://www.wipo.int/edocs/pubdocs/es/intproperty/941/wipo_pub_941_2010.pdf

Piñeiro, L. (2015). Argentina inagura el tercer radar primario 3D de largo alcance y firma contrato para la construcción de seis nuevos radares. defensa.com. https://www.defensa.com/argentina/argentinainaugura-tercer-radar-primario-3d-largo-alcance-firma

Potenze, P. L. (2017). Los radares argentinos. Gaceta Aeronautica. https:/ /www.gacetaeronautica.com/gaceta/wp-101/?p=22386

Prime Faraday. (2002). An Introduction to MEMS (Micro-electromechanical Systems)—An-introduction-tomems.pdf. Prime Faraday Technology Watch. http://www.lboro.ac.uk/microsites/mechman/research/ipm$\mathrm{ktn} / \mathrm{pdf} /$ Technology_review/an-introduction-to-mems.pdf

Quiroga, J. M. (2018). Políticas públicas, trayectorias institucionales y desarrollo tecnológico nacional. Los primeros sesenta años de tecnología radar en la Argentina. En Políticas de ciencia, tecnología e innovación en la Argentina de la postdictadura. Editorial UNRN.

Quiroga, J. M. (2020). Capacidades dinámicas en la producción de bienes intensivos en conocimientos. El caso del desarrollo de radares en Argentina (2003-2015). CTS - Revista Iberoamericana de Ciencia, Tecnología y Sociedad. https://doi.org/10.13140/RG.2.2.27266.63680

Quiroga, J. M., \& Aguiar, D. (2016). Abriendo la "caja negra" del radar. Las políticas de radarización para uso civil y de defensa en Argentina entre 1948 y 2004. H-Industri@: Revista De Historia De La Industria, Los Servicios Y Las Empresas En América Latina, 19, 71-100.

Sábato, J. A. (2004). Ensayos en campera (1a ed.). Bernal: Univ. Nacional de Quilmes Editorial.

Susskind, C. (1994). Radar as a case study in simultaneous invention. En Tracking the history of radar. IEEE-Rutgers Center for the History of Electrical Engineering and the Deutsches Museum.

Toledo, D. G. C. (2019). Aspectos históricos e conceituais da dependência tecnológica da América Latina sob o novo neocolonialismo. OIKOS, 18(3), 41-56.

Vasconcelos, Y. (2013). De misiles a radares. Pesquisa Fapesp, 202. https://revistapesquisa.fapesp.br/es/de-misiles-radares/

Vassallo, C. M. (2008). Control del espácio aéreo en la República Argentina [Ministerio de Justicia y Derechos Humanos Argentina]. SAIJ. http://www.saij.gob.ar/doctrina/dacf080092-vassallocontrol_espacio_aereo_en.htm\%3Bjsessionid=el1a0negeh3fppq327uq3y5e?0 